\title{
Pulmonary edema during cesarean delivery in a patient with a mediastinal mass
}

\section{Edème pulmonaire pendant un accouchement par césarienne chez une patiente présentant une masse médiastinale}

\author{
Uma Shastri, MD • Peter Slinger, MD • \\ Elsie Nguyen, MD • Jose C. A. Carvalho, MD, PhD • \\ Mrinalini Balki, MD \\ Received: 5 August 2010/Accepted: 15 November 2010/Published online: 4 December 2010 \\ (C) Canadian Anesthesiologists' Society 2010
}

\begin{abstract}
Purpose To report the management of a Cesarean delivery complicated by postpartum pulmonary edema in a parturient with a large mediastinal mass.

Clinical findings A patient with an anterior mediastinal mass presented to the labour and delivery floor at 37 weeks of gestation with cough, severe dyspnea, and chest pain. Radiological investigations revealed that the mass occupied mainly the right side of the thorax and produced compression of the mediastinal structures. The patient underwent an elective Cesarean delivery under epidural anesthesia; however, immediately following the placental delivery, she developed unilateral pulmonary edema. She was managed successfully with diuretics, and her subsequent course in the hospital was uneventful. The biopsy of the mass revealed a lymphoma, which was treated eventually by chemotherapy. Both the mother and her baby are doing well after two years of follow-up.

Conclusion A multidisciplinary approach should be considered in the management of a pregnant patient with symptomatic anterior mediastinal mass. Practitioners
\end{abstract}

U. Shastri, MD · J. C. A. Carvalho, MD, PhD · M. Balki, MD ( $ه)$

Department of Anesthesia and Pain Management, Mount Sinai

Hospital, University of Toronto, 600 University Avenue,

Room 1514, Toronto, ON M5G 1X5, Canada

e-mail:mrinalini.balki@uhn.on.ca

P. Slinger, MD

Department of Anesthesia and Pain Medicine, Toronto General Hospital, University of Toronto, 200 Elizabeth Street,

Toronto, ON M5G 2C4, Canada

E. Nguyen, MD

Department of Medical Imaging, Toronto General Hospital,

University of Toronto, Toronto, ON, Canada should be aware of the possibility of unilateral pulmonary edema in patients with an anterior mediastinal mass compressing pulmonary veins. Such patients should be treated promptly with diuretics to prevent further adverse outcomes.

\section{Résumé}

Objectif Rapporter la prise en charge d'un accouchement par césarienne compliqué par un odème pulmonaire post-partum chez une parturiente présentant une importante masse médiastinale.

Constatations cliniques Une patiente avec une masse médiastinale antérieure s'est présentée à la salle d'accouchement à 37 semaines de gestation. Elle toussait, présentait une dyspnée grave et se plaignait de douleur thoracique. Des examens radiologiques ont révelé qu'une masse occupait principalement le côté droit du thorax et provoquait une compression des structures médiastinales. La patiente a subi une césarienne non urgente sous anesthésie péridurale; toutefois, immédiatement après la délivrance placentaire, elle a manifesté un cedème pulmonaire unilatéral. La patiente a été traitée avec des diurétiques et aucune autre complication n'a été notée durant son séjour à l'hôpital. La biopsie de la masse a révelé un lymphome, pour lequel on a administré une chimiothérapie par la suite. Après deux ans de suivi, la mère et l'enfant vont bien.

Conclusion Une approche pluridisciplinaire devrait être envisagée pour la prise en charge d'une patiente enceinte présentant une masse médiastinale antérieure symptomatique. Les praticiens doivent être conscients qu'un xedème pulmonaire unilatéral est une complication possible chez les patients présentant une masse médiastinale antérieure qui comprime les veines pulmonaires. Il convient de traiter de tels patients dans les 
plus brefs délais à l'aide de diurétiques afin de prévenir d'autres complications.

The perioperative management of a patient with an anterior mediastinal mass can be associated with severe lifethreatening complications and anesthetic challenges due to its proximity to various airway and vascular structures and the potential to cause their compression. Pregnancy can make the diagnosis of a growing intrathoracic mass more difficult, as the symptoms produced by the tumour may mimic those arising from the normal physiologic changes of pregnancy and, hence, may not be investigated actively. We report the anesthetic management for Cesarean delivery $(\mathrm{CD})$ in a patient with severe respiratory symptoms associated with a large anterior mediastinal tumour. Written consent for publication of this report was obtained from the patient.

\section{Case description}

At 37 weeks of gestation, a 28-yr-old primigravida presented to the labour and delivery floor with two months' history of non-productive cough without hemoptysis. She also described symptoms of orthopnea, dyspnea, and chest pain. Her symptoms had worsened progressively, especially over the previous two weeks, and they were exacerbated when she was lying on her left side. At the time of presentation, the patient's orthopnea was so significant that she was unable to lie supine and required a $45^{\circ}$ head-up position. She also reported night sweats, decreased appetite, and a $2.3 \mathrm{~kg}$ weight loss over a period of three weeks. Her medical history was significant for hypothyroidism that was well controlled with levothyroxine $0.15 \mathrm{mg}$ daily. Fetal growth was appropriate for the gestational age.

On physical examination, the patient's height and weight were $160 \mathrm{~cm}$ and $75.9 \mathrm{~kg}$ respectively, and her vital signs were as follows: blood pressure $115 / 70 \mathrm{mmHg}$, heart rate 120 beats $\cdot \mathrm{min}^{-1}$, respiratory rate 20 breaths $\cdot \mathrm{min}^{-1}$, and oxygen saturation $\left(\mathrm{SpO}_{2}\right) 92 \%$ on room air. There was no clinical evidence of superior vena cava syndrome or respiratory stridor; jugular venous pressure was at $3 \mathrm{~cm}$ above sternal angle, and there was no pedal edema. Her heart sounds were normal on auscultation and no murmurs were heard. Air entry was reduced significantly in all lobes of the right lung; however, air entry was normal on the left side, and no crackles or wheezes were heard. Evaluation of the patient's airway revealed normal parameters with a Mallampati class 2 view. The thoracolumbar spine appeared normal on palpation.

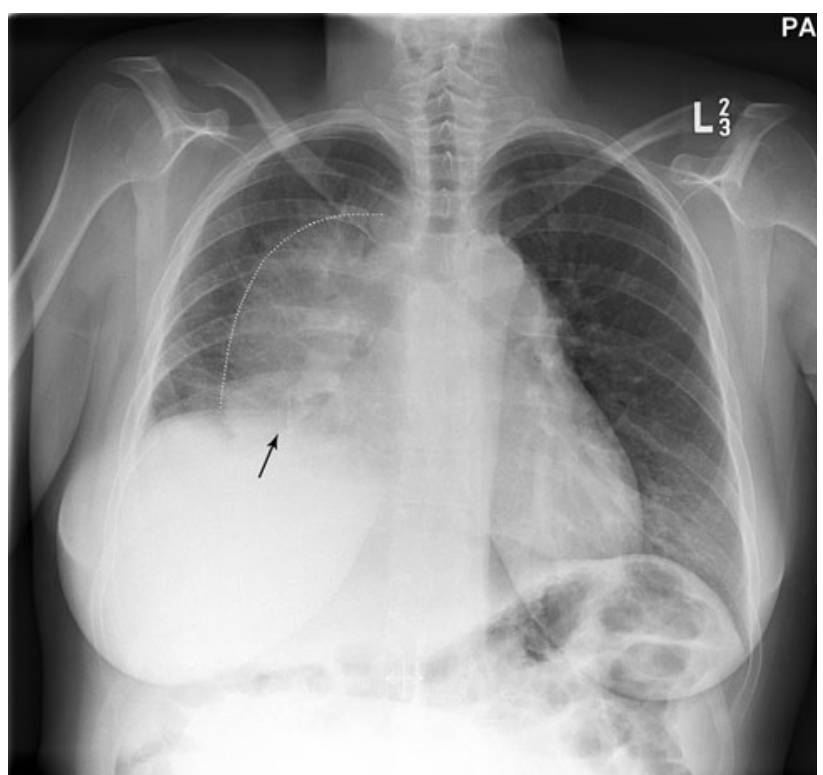

Fig. 1 Preoperative frontal chest radiograph shows right anterior mediastinal mass (black arrow) extending approximately $15 \mathrm{~cm}$ superior-anteriorly (outlined)

The patient's initial chest $x$-ray revealed a large anterior mediastinal mass extending superior-anteriorly (Fig. 1). A magnetic resonance imaging (MRI) was also done; however, it was limited by the patient's inability to lie supine due to her shortness of breath. It showed a large mass $(17 \mathrm{~cm} \times 8 \mathrm{~cm} \times 15 \mathrm{~cm})$ occupying the anterior mediastinum, which compressed most of her right lung and encased the right superior pulmonary vein. In addition, there was compression of the right upper and middle lobe bronchi, right lung atelectasis, and a right pleural effusion. There were no conduction abnormalities noted on the electrocardiogram, and the transthoracic echo revealed normal right and left ventricular function. Although there was some compression of the right ventricular outflow tract (RVOT), there was no RVOT gradient. Pulmonary function tests showed a restrictive lung disease pattern with forced vital capacity (FVC) $1.58 \mathrm{~L}$ ( $42 \%$ of predicted), forced expiratory volume in $1 \mathrm{sec}\left(\mathrm{FEV}_{1}\right)$ 1.39L (47\% of predicted), $\mathrm{FEV}_{1} / \mathrm{FVC}$ ( $88 \%$ of predicted), peak expiratory flow rate $3.43 \mathrm{~L}$ ( $54 \%$ of predicted), vital capacity $1.68 \mathrm{~L}$ ( $42 \%$ of predicted), total lung capacity $2.59 \mathrm{~L}$ (52\% of predicted), and residual volume $1.02 \mathrm{~L}$ (69\% of predicted). The decreases in the values from the predicted reference range indicated severe disease and were consistent with the patient's limited functional capacity. The differential diagnosis of the mass included lymphoma and thymoma and was less likely a retrosternal thyroid mass or teratoma.

The patient was admitted to the high-risk obstetric service. Anesthesia, respirology, oncology, and thoracic surgery were consulted, and after discussion with the 
patient, an elective $\mathrm{CD}$ was planned for the next day. Additional staff personnel, including a thoracic anesthesiologist and a thoracic surgeon, were present for the surgery, and emergency airway equipment, including a rigid bronchoscope and difficult airway cart, were kept on standby. In the operating room, two $16 \mathrm{G}$ intravenous cannulae were sited, one in each forearm vein. An arterial line was inserted in the right radial artery and standard monitors were applied. The patient's vital signs were stable, and $\mathrm{SpO}_{2}$ increased from $92 \%$ to $97 \%$ on $8 \mathrm{~L} \cdot \mathrm{min}^{-1}$ oxygen by face mask. Lumbar ultrasound scanning was performed to determine the epidural needle insertion point at the L3-4 interspace in the sitting position, and the epidural space was identified at $4.5 \mathrm{~cm}$. A 19G epidural catheter was inserted via a $17 \mathrm{G}$ Touhy needle in the usual manner at this level. The position of the catheter was confirmed by its electrical stimulation, and the patient was positioned supine with a slight right lateral tilt, and her back was elevated to $30^{\circ}$, as this was the only position she could tolerate. Thereafter, incremental 5 -mL boluses of lidocaine $2 \%$ with 1:200,000 epinephrine and fentanyl $100 \mu \mathrm{g}$ were administered slowly through the epidural catheter to achieve a T4 sensory block; a total of $20 \mathrm{~mL}$ was injected. Fluid administration consisted of Ringer's lactate $1 \mathrm{~L}$ and pentaspan $250 \mathrm{~mL}$ given during the perioperative period. An attempt was made to maintain blood pressure at baseline levels by using phenylephrine in 100 $\mu \mathrm{g}$ aliquots. The patient received a total of $1.5 \mathrm{mg}$ of phenylephrine throughout the procedure. A male infant, with an Apgar score of 9 at one minute and 9 at five minutes, was delivered uneventfully, and an infusion of oxytocin (20 IU. $\mathrm{L}^{-1}$, initial rapid infusion of $500 \mathrm{mU}$ followed by $40 \mathrm{mU} \cdot \mathrm{min}^{-1}$ ) was started. The patient tolerated the beginning of surgery until the delivery of the infant very well; however, immediately after the delivery of the placenta, she developed severe cough, shortness of breath, and desaturation to $93 \%$. She also had new onset audible crackles mainly in the right lung fields. She was treated with oxygen supplementation, furosemide $20 \mathrm{mg} i v$ and was positioned even more upright with a right-sided tilt. Her postoperative chest $x$-ray and computed tomography (CT) scan showed increased opacification of the right hemithorax due to new pulmonary edema as well as a moderately large right-sided pleural effusion (Figs. 2 and 3). Her CT scan also revealed significant encasement by the mass and complete occlusion of the right superior pulmonary vein with less severe compression of the right inferior pulmonary vein. However, the right upper lobe pulmonary artery was encased and obstructed distally (Fig. 4). The findings on the CT scan were consistent with that of the initial MRI; however, the CT scan provided some additional information. It showed an approximate $50 \%$ narrowing of the right middle lobe bronchus

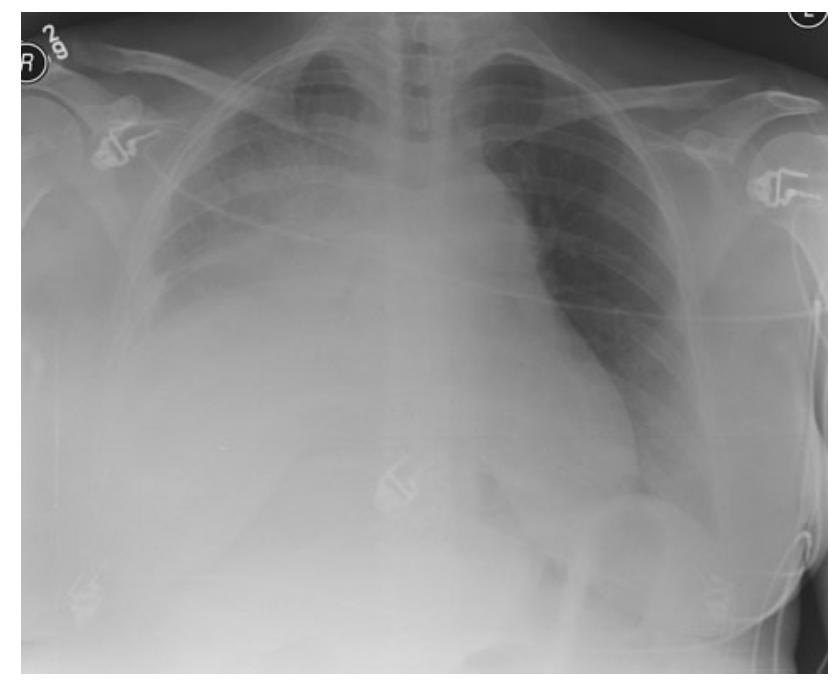

Fig. 2 Postoperative anteroposterior portable chest radiograph demonstrates increased opacification of the right hemithorax, due, in part, to the anterior mediastinal mass but also due to the recent moderately large right pleural effusion and the recent right upper lobe opacity consistent with pulmonary edema. The left lung remained clear

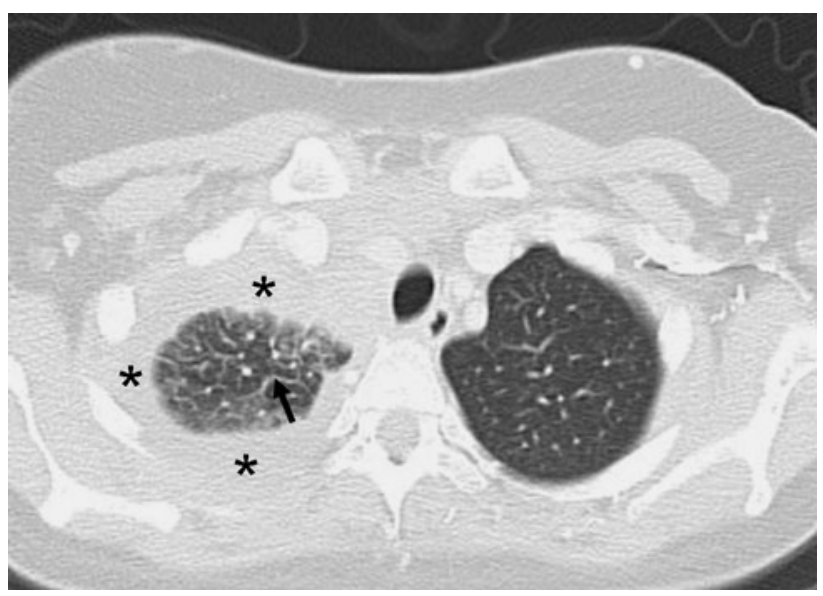

Fig. 3 Postoperative axial computed tomography scan in lung windows shows pulmonary edema characterized by interlobular septal thickening (black arrow), right pleural effusion (asterisks), and some ground glass density. Note the left upper lobe, which is normal with no evidence of pulmonary edema or pleural effusion

proximally and compressive atelectasis of the medial and lateral segments of the entire right middle lobe due to the mediastinal mass. The superior segment right lower lobe bronchi were mildly narrowed but still patent. The mass did not compress the patient's trachea but was causing superior vena cava obstruction below the level of the azygous vein confluence, resulting in chest wall and mediastinal venous collaterals and dilatation of the azygous and hemiazygous veins. The mass was in close contact with the ascending aorta, main pulmonary artery, and right pulmonary artery, obliterating the intervening fat planes 


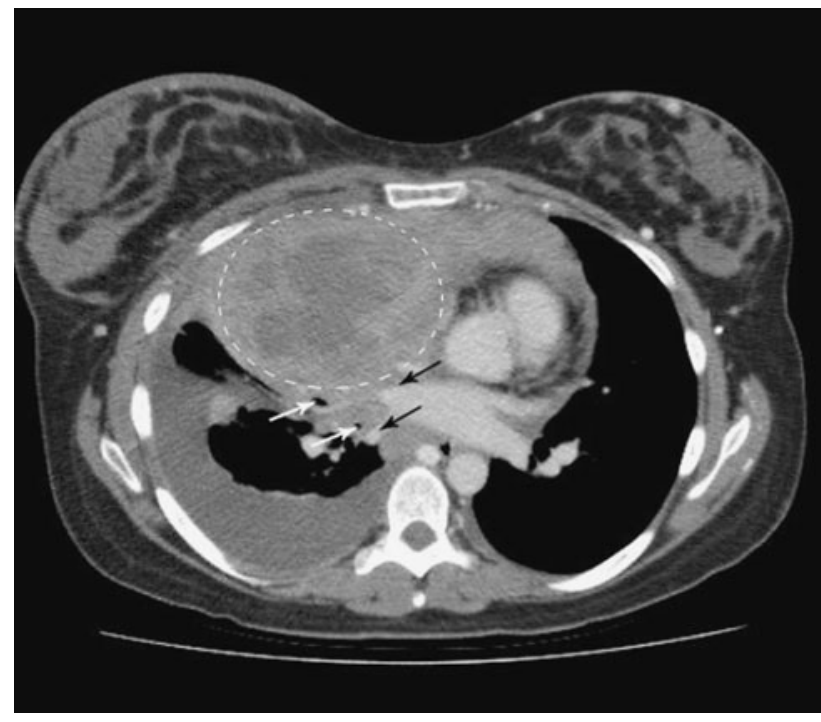

Fig. 4 Postoperative computed tomography scan shows a large anterior mediastinal mass (outlined) compressing and encasing the right superior and, to a lesser extent, the inferior pulmonary veins (black arrows) and the right middle and lower lobe bronchi (white arrows). A moderately large right pleural effusion is also seen

but not causing compression of these vascular structures. There was a small pericardial effusion with no distortion of cardiac anatomy. Mild leftward mediastinal shift was seen due to the large anterior mediastinal mass.

The patient was treated with additional diuretics and transferred to the intensive care unit (ICU) for continuous monitoring with all emergency airway equipment in close proximity. During the ensuing eight hours in the ICU, her dyspnea improved dramatically, $\mathrm{SpO}_{2}$ increased to $95 \%$ on room air, and no crackles were audible on auscultation. On the fourth postoperative day, she underwent biopsy of the mass under local anesthesia, which confirmed a non-B-cell lymphoma. Subsequently, the size of her tumour decreased markedly with chemotherapy. On long-term follow-up after two years, both the mother and her baby were doing well.

\section{Discussion}

Our patient developed unilateral pulmonary edema immediately following the delivery; however, immediate diagnosis and prompt treatment led to an early resolution of her symptoms and a successful outcome.

After urgent multi-disciplinary team discussions, elective $\mathrm{CD}$ was chosen over either waiting for the patient to go into spontaneous labour or performing an induction. This option was preferred to prevent further deterioration of her respiratory status and to avoid any possible airway intervention should she present with any major obstetric complication or require emergency $\mathrm{CD}$ during labour. Since the procedure was amenable to regional technique, an epidural was selected over a spinal to allow careful titration of drugs, better hemodynamic stability, and supplementation of block, if required. A previous case resulting in a successful outcome has been described wherein a patient with a mediastinal mass underwent a CD with continuous spinal anesthesia using a subarachnoid catheter. $^{1}$

It is well recognized that the induction of general anesthesia in a patient with a large mediastinal mass could be associated with high morbidity and mortality due to fatal airway obstruction or cardiovascular collapse. ${ }^{2,3}$ In our case, contingency planning for securing the airway and converting to general anesthesia for any unexpected situation included availability of extra anesthesiologists and a thoracic surgeon as well as adequate resources, such as a difficult airway cart that included a rigid bronchoscope, jet ventilator, and double lumen endotracheal tubes. If an unexpected situation were to happen, our plan was as follows: a) to reposition the patient to alleviate airway obstruction; b) to secure the airway by placing a single/ double lumen endotracheal tube with a fibreoptic intubation while the patient is fully awake and spontaneously breathing; c) to stent the airway using rigid bronchoscopy and ventilation via venturi jet in case of airway collapse; and if ultimately necessary, d) to perform an immediate sternotomy by the thoracic surgeon and elevate the mass from the major cardiovascular structures to relieve compression.

Although the establishment of cardiopulmonary bypass by femoral cannulations prior to induction of general anesthesia has been performed safely in adult patients with mediastinal masses, ${ }^{4}$ it has not been reported in pregnant patients. The usefulness of femorofemoral bypass "on standby" is considered to be uncertain. ${ }^{3}$ Even with a primed pump and a prepared team, it should be recognized that cannulating and establishing adequate circulation and oxygenation once the airway or cardiovascular collapse has occurred would require at least five to ten minutes. This course of action may resuscitate the patient but with possible neurological injury. ${ }^{5,6}$ It is evident that general anesthesia cannot be given safely to patients with severe positional symptoms, even with maintenance of spontaneous ventilation, unless an alternative technique to maintain oxygenation or circulation, such as cardiopulmonary bypass, has been established pre-emptively. ${ }^{3}$

The development of right-sided pulmonary edema in our patient immediately after the delivery was likely due to the autotransfusion of blood caused by uterine contraction, leading to a sudden increase in preload. Healthy parturients can usually compensate for the additional blood volume from the contracting uterus by increasing cardiac output. 
In our case, however, the predominantly right-sided pulmonary edema occurred secondary to the increased pulmonary capillary pressure caused by the right superior pulmonary vein compression that led to impaired forward flow. The symptoms followed sequentially after the satisfactory uterine tone was noted by the obstetrician. In anticipation of this problem, one may consider judicious administration of intravenous fluids, aggressive use of vasopressors for treating any hypotension, and close hemodynamic monitoring. However, despite these measures, such patients may develop pulmonary edema, as seen in our case. When administered in large boluses, oxytocin also has the potential to worsen pulmonary edema in the presence of obstruction to forward flow, perhaps due to its antidiuretic effect; however, this scenario is unlikely if oxytocin is administered in the form of a slow infusion. ${ }^{7}$

A multidisciplinary approach is imperative in the management of pregnant patients with anterior mediastinal masses. Although these cases have been managed successfully with spinal, epidural, and general anesthesia, ${ }^{1,8-11}$ the most important factor in choosing a technique should be patient safety, as each of these techniques requires specific planning to deal with respective associated complications. One should be aware of the possibility that patients with an anterior mediastinal mass compressing pulmonary veins could develop pulmonary edema immediately following the delivery. This likelihood should be anticipated, and this complication should be treated urgently to prevent further respiratory and cardiovascular compromise. An attempt should be made to prevent this complication in such parturients by using prophylactic diuretics after the delivery in the presence of close hemodynamic monitoring.
Conflicts of interest None declared.

\section{References}

1. Martin WJ. Cesarean section in a pregnant patient with an anterior mediastinal mass and failed supradiaphragmatic irradiation. J Clin Anesth 1995; 7: 312-5.

2. Gothard JW. Anesthetic considerations for patients with anterior mediastinal masses. Anesthesiol Clin 2008; 26: 305-14.

3. Slinger P, Karslib $C$. Management of the patient with a large anterior mediastinal mass: recurring myths. Curr Opin Anaesthesiol 2007; 20: 1-3.

4. Tempe DK, Arya $R$, Dubey $S$, et al. Mediastinal mass resection: femorofemoral cardiopulmonary bypass before induction of anesthesia in the management of airway obstruction. J Cardiothoracic Vasc Anesth 2001; 15: 233-6.

5. Takeda S, Miyoshi S, Omori K, Okumura M, Matsuda H. Surgical rescue for life-threatening hypoxemia caused by a mediastinal tumor. Ann Thorac Surg 1999; 68: 2324-6.

6. Turkoz A, Gulcan O, Tercan F, Kocum T, Turkoz R. Hemodynamic collapse caused by a large unruptured aneurysm of the ascending aorta in an 18 year old. Anesth Analg 2006; 102: 1040-2.

7. Ghai B, Vayjnath AM, Lal S. Acute pulmonary oedema following oxytocin administration: a life threatening complication. J Indian Med Assoc 2006; 104: 261-2.

8. Dasan J, Littleford J, McRae K, Farine D, Winton T. Mediastinal tumour in a pregnant patient presenting as acute cardiorespiratory compromise. Int J Obstet Anesth 2002; 11: 52-6.

9. Crosby E. Clinical case discussion: anesthesia for cesarean section in a parturient with a large intrathoracic tumour. Can J Anesth 2001; 48: 575-83.

10. Buvanendran A, Mohajer P, Pombar X, Tuman KJ. Perioperative management with epidural anesthesia for a parturient with superior vena caval obstruction. Anesth Analg 2004; 98: 1160-3.

11. Webster JA, Self $D D$. Anesthesia for pericardial window in a pregnant patient with cardiac tamponade and mediastinal mass. Can J Anesth 2003; 50: 815-8. 